\title{
Crossing number and diameter of boundary slope set of Montesinos knot
}

\author{
Kazuhiro ICHihara AND Shigeru Mizushima
}

It is shown that the diameter of the boundary slope set is bounded from above by the twice of the minimal crossing number for a Montesinos knot.

\section{Introduction}

For a knot $K$ in the 3 -sphere $S^{3}$, the minimum number of crossings of a diagram among all diagrams of $K$ is called the crossing number. This is one of the most basic topological complexities of a knot.

Another complexity of knots which we consider in this paper is the diameter of the set of boundary slopes. Let $E(K)$ be the exterior of $K$. A compact connected surface properly embedded in $E(K)$ is said to be essential if the surface is incompressible and boundary-incompressible. The boundary of an essential surface appears on $\partial E(K)$ as a parallel family of non-trivial simple closed curves, and so, it determines an isotopy class of the curves, which is called the boundary slope of the surface. Recall that the set of slopes is identified with the set of rational numbers with $\infty$, where the meridian of $K$ corresponds to $\infty$, by using the standard meridian-longitude system for $K$. Then the diameter of the set of boundary slopes for $K$ is defined as the difference between the greatest and the least ones as rational numbers, except for the infinity (i.e., meridional) slope. This is well-defined since it is known that there are only finitely many boundary slopes [4] and there exist at least two boundary slopes in general [2].

Our main result is the following.

Theorem 1.1 (main theorem). Let $K$ be a Montesinos knot without 1/0-tangles. Then, we have

$$
2 \operatorname{cr}(K) \geq \operatorname{Diam}(K),
$$


where $\operatorname{cr}(K)$ denotes the crossing number of $K$ and $\operatorname{Diam}(K)$ denotes the diameter of the set of boundary slopes for $K$. The equality holds if $K$ is alternating.

It is probable that this inequality holds for all knots in general. For example, it is easily verified for torus knots. For a non-trivial torus knot $T_{p, q}$, assuming that $p, q$ are relatively prime with $2 \leq q \leq p$, it is known that the boundary slopes are 0 and $p q$ while the crossing number is $p q-p$. Thus, we have $2 \operatorname{cr}\left(T_{p, q}\right)=p q+p(q-2) \geq p q=\operatorname{Diam}\left(T_{p, q}\right)$. The equality holds when $q=2$, equivalently, the knot is alternating. Moreover, as far as the authors observed, the equality holds for all alternating knots. We here remark that, for alternating knots, the converse inequality $\operatorname{Diam}(K) \geq$ $2 \mathrm{cr}(K)$ can be shown. By the checkerboard construction for the reduced alternating diagram, we can obtain two surfaces, which are both essential by $[1,3]$, and the difference between their boundary slopes is actually $2 \operatorname{cr}(K)$.

To prove the theorem, we mainly use the algorithm given by Hatcher and Oertel in [5], which enumerates all boundary slopes for a given Montesinos knot. The algorithm in turn is based on the algorithm by Hatcher and Thurston in [6], which enumerates all boundary slopes for a two-bridge knot. Another ingredient is the way to calculate the crossing number of a given Montesinos knot obtained by Lickorish and Thistlethwaite in [9]. In the next section, we introduce some notation and terminology. Then, we prove the main theorem in the last section.

The authors would like to thank Masaharu Ishikawa for suggesting the potential relationship between the crossing number and the diameter of the set of boundary slopes. They also thank Thomas Mattman for letting them know the work [10]. They are grateful to the referee for careful reading of the manuscript and useful comments

\section{Preliminary}

In this section, we prepare various notations and recall fundamentals.

We start with a rational tangle, which we regard as follows (see figure 1). The left figure illustrates a sphere with four punctures, which is flattened like a "pillowcase." Consecutive segments with slope $1 / 2$ are drawn from each of the four punctures. These segments form two arcs. Think about the interior of the sphere, and push these two arcs into the interior with four ends fixed. See the right diagram in figure 1 . Then, we have a $1 / 2$-tangle in a 3 -ball. Similarly, we can obtain a $p / q$-tangle for arbitrary irreducible fraction $p / q$ including $1 / 0$. 

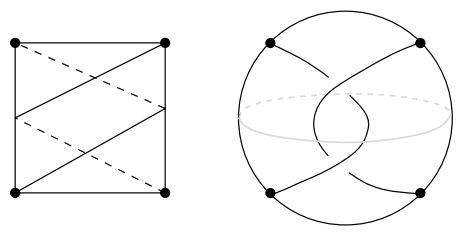

Figure 1: Arcs on a pillowcase and 1/2-tangle.

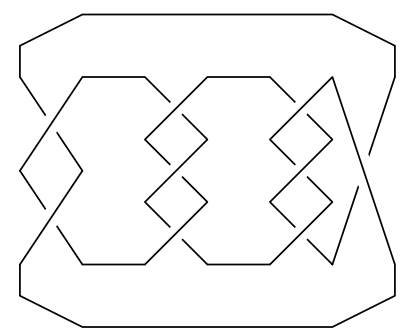

Figure 2: A diagram of a Montesinos knot $K(1 / 2,1 / 3,-2 / 3)$.

A Montesinos knot is defined as a knot obtained by putting rational tangles together in a circle (see figure 2). A Montesinos knot obtained from rational tangles $R_{1}, R_{2}, \ldots, R_{N}$ is denoted by $K\left(R_{1}, R_{2}, \ldots, R_{N}\right)$. The number of tangles will be denoted by $N$ throughout this paper. Concerning such a tuple $\left(R_{1}, R_{2}, \ldots, R_{N}\right)$, we will keep the following assumptions:

- In general, by combining rational tangles, we have a Montesinos link with one or more link components. However, in this paper, we want to only consider Montesinos knots; the number of components is one. Thus, we assume that the tuple must satisfy either of the two conditions: (a) exactly one of the denominators of $R_{i} \mathrm{~s}$ is even or (b) all denominators of $R_{i} \mathrm{~s}$ are odd and the number of odd numerators is odd.

- We always assume that none of the $R_{i}$ s are $1 / 0$. Furthermore, we always assume $N \geq 3$ since if the number of tangles is two or less, the knot is found to be a two-bridge knot. For the two-bridge knots, a result corresponding to the main theorem in this paper has been presented by Mattman et al. in [10].

- We assume that each tangle is non-integral, since an integral tangle can be combined with an adjacent rational tangle. This operation is a kind of normalization. 


\subsection{Boundary slopes for Montesinos knot}

In this subsection, we review the notions developed in the work of Hatcher and Oertel [5] about the boundary slopes for Montesinos knots. Though we will try to give necessary explanations so that this paper can be selfcontained, it would be more preferable that the reader is rather familiar to their machinery. Also, see our previous note [7] for detail.

Throughout the following, let $K$ be a Montesinos knot obtained from rational tangles $R_{1}, R_{2}, \ldots, R_{N}$. In sequel, $R_{i}$ will denote a fraction or the corresponding rational tangle depending on the situation.

We first give an outline of the machinery developed by Hatcher and Oertel briefly.

Take a simple unknotted loop in the 3 -sphere $S^{3}$, and set disjoint $N$ disks bounded by the loop. By the disks, $S^{3}$ is divided into $N$ 3-balls. After appropriate isotopies, $K$ is divided into rational tangles $\left(R_{1}, R_{2}, \ldots, R_{N}\right)$, the intersections with the $N$ balls. Let $F$ be an essential (meaning that, incompressible and boundary-incompressible) surface properly embedded in the exterior of $K$. By virtue of Proposition 1.1 in [5], $F$ can be isotoped so that it is divided into subsurfaces $\left(F_{1}, F_{2}, \ldots, F_{N}\right)$ in certain "good position" included in each of the $N$ balls. Then each $F_{i}$ is represented by an "edgepath" $\gamma_{i}$ in certain "diagram" $\mathcal{D}$, and the whole $F$ is represented by an "edgepath system" $\Gamma$ in $\mathcal{D}$. Actually the boundary slope of $F$ is calculated from the corresponding $\Gamma$ in a purely combinatorial way. Conversely an "edgepath system" $\Gamma$ in $\mathcal{D}$ corresponds to a properly embedded surface in the exterior of $K$. In [5], conditions for determining essentiality of the surface are fully described.

In the following nine subsubsections, we will summarize the features of the diagram $\mathcal{D}$, edges, edgepaths and edgepath systems in $\mathcal{D}$, which we will use in the rest of the paper.

2.1.1. Diagram. The diagram $\mathcal{D}$ is described as the 1 -skeleton of a triangulation of a region on a plane as illustrated in figure 3. Precisely a vertex of $\mathcal{D}$ indicates either a point $(u, v)=((q-1) / q, p / q)$ denoted by $\langle p / q\rangle$, a point $(u, v)=(1, p / q)$ denoted by $\langle p / q\rangle^{\circ}$ where $p / q$ is an irreducible fraction, or a point $(u, v)=(-1,0)$ denoted by $\langle 1 / 0\rangle=\langle\infty\rangle$. See also figure 4 .

2.1.2. Edges. Two vertices $\langle p / q\rangle$ and $\langle r / s\rangle$ in $\mathcal{D}$ are connected by an edge if $|p s-q r|=1$. Such an edge is denoted by $\langle p / q\rangle-\langle r / s\rangle$. An important class of the edges are the vertical edges, which connect the vertices $\langle z\rangle$ and $\langle z+1\rangle$ for arbitrary integer $z$. Another important class of the edges are 


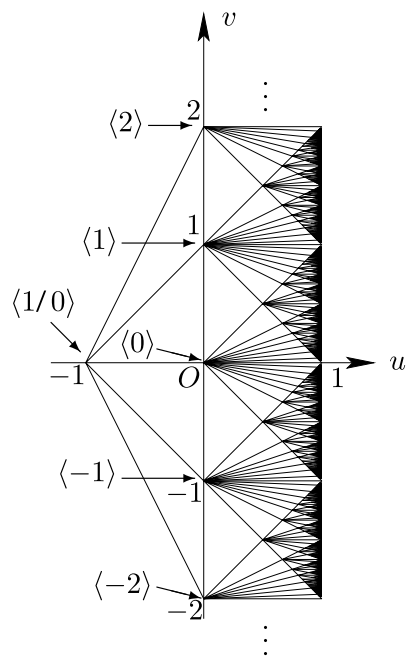

Figure 3: The diagram $\mathcal{D}$.

the $\infty$-edges, which connect the vertices $\langle\infty\rangle$ and $\langle z\rangle$ for integer $z$. There is another kind of edge called a horizontal edge, which connects $\langle p / q\rangle$ and $\langle p / q\rangle^{\circ}$ for each $p / q$.

Let $e$ be an edge $\langle p / q\rangle-\langle r / s\rangle$ with $q \geq 1, s \geq 1$. Let $k$ and $m$ be integers satisfying $m \geq 2$ and $1 \leq k \leq m-1$. Then, let $\frac{k}{m}\langle p / q\rangle+\frac{m-k}{m}\langle r / s\rangle$ denote a point on $e$ with $u v$-coordinates $(u, v)=\frac{k q}{k q+(m-k) s}\left(\frac{q-1}{q}, \frac{p}{q}\right)+\frac{(m-k) s}{k q+(m-k) s}\left(\frac{s-1}{s}, \frac{r}{s}\right)$. This is called a rational point of the edge $e$. For an $\infty$-edge $\langle 1 / 0\rangle-\langle z\rangle$, let $\frac{k}{m}\langle 1 / 0\rangle+\frac{m-k}{m}\langle z\rangle$ denote a point with $(u, v)=\frac{k}{m}(-1,0)+\frac{m-k}{m}(0, z)$ on the edge, which is a rational point of the $\infty$-edge. A partial edge is an edge $\left(\frac{k}{m}\langle p / q\rangle+\frac{m-k}{m}\langle r / s\rangle\right)-\langle r / s\rangle$. On the other hand, a non-partial edge is called a complete edge.

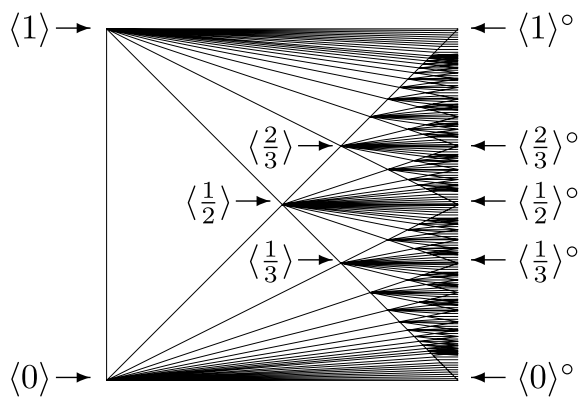

Figure 4: A part of the diagram $\mathcal{D}$ in $[0,1] \times[0,1]$. 
Let $e$ be a horizontal edge $\langle p / q\rangle-\langle p / q\rangle^{\circ}$. Then, for integers $l \geq 1$ and $m \geq 1$ let $\frac{m}{m+l}\langle p / q\rangle+\frac{l}{m+l}\langle p / q\rangle^{\circ}$ denote a point with $(u, v)=\frac{m}{m+l}\left(\frac{q-1}{q}, \frac{p}{q}\right)+$ $\frac{l}{m+l}\left(1, \frac{p}{q}\right)$ on the edge $e$. This is a rational point of the horizontal edge.

Assume that a non- $\infty$-edge is oriented from right to left. Then it is said to be increasing or decreasing if the $v$-coordinate increases or decreases as a point moves along the edge in that direction. That is, an edge in the region $u>0$ is increasing or decreasing if the $v$-coordinate increases or decreases as the $u$-coordinate decreases.

We assign +1 or -1 to an edge $e$ according to whether $e$ is increasing or decreasing, respectively. This is called the sign of the edge $e$, and is denoted by $\sigma(e)$.

The length of a complete edge is set to be 1 . The length of a partial edge $\left(\frac{k}{m}\langle p / q\rangle+\frac{m-k}{m}\langle r / s\rangle\right)-\langle r / s\rangle$ is set to be $\frac{k}{m}$. Let $|e|$ denote the length of an edge $e$.

2.1.3. Edgepaths. Roughly, an edgepath is a connected piecewise linear path in the diagram $\mathcal{D}$. There are two kinds of edgepaths; non-constant and constant edgepaths.

The first one is a path on $\mathcal{D}$ starting from a vertex $\langle R\rangle$. We will only consider such an edgepath with the following two conditions:

(1) It must run from right to left in the weak sense, where vertical edges are allowed.

(2) It must be minimal, that is, it must not retrace back nor include two successive edges on a common triangle.

We call an edgepath of this kind a non-constant edgepath. A non-constant edgepath is expressed by a sequence of vertices like $\left\langle p_{k} / q_{k}\right\rangle-\left\langle p_{k-1} / q_{k-1}\right\rangle-$ $\cdots-\left\langle p_{2} / q_{2}\right\rangle-\left\langle p_{1} / q_{1}\right\rangle$, where $p_{1} / q_{1}=R$. Note here that the vertices are listed from right to left so that the direction of the sequence coincides with the direction of an edgepath in the diagram $\mathcal{D}$. Also note that we allow that the last edge can be a partial edge.

An edgepath of the second kind is a single point on a horizontal edge $\langle R\rangle-\langle R\rangle^{\circ}$. This is called a constant edgepath. The single point of a constant edgepath must be a rational point of the horizontal edge $\langle R\rangle-\langle R\rangle^{\circ}$.

We will always use the symbol $\gamma$ for edgepaths. In the following, we collect some terminologies about edgepaths.

Assume that the $u$-coordinate of the left endpoint of an edgepath is $u_{0}$. Then the edgepath is said to be of type I, type II or type III according to which of $u_{0}>0, u_{0}=0$ or $u_{0}<0$ the coordinate satisfies. 
We here introduce a new notion: a basic edgepath, which is not used in [5]. An edgepath is called a basic edgepath if the edgepath ends at the point with $u$-coordinate 0 and includes no vertical edges. We usually use symbol $\lambda$ for basic edgepaths.

An edgepath is said to be monotonically increasing (respectively monotonically decreasing), if all non- $\infty$-edges in the edgepath are increasing (resp. decreasing). We ignore $\infty$-edges when we judge an edgepath is monotonic or not. For the vertex $\langle p / q\rangle(q \geq 2)$, there is one increasing leftward edge and one decreasing leftward edge. Hence, there exists just one monotonically increasing basic edgepath and just one monotonically decreasing basic edgepath for a fixed fraction $p / q(q \geq 2)$.

The length of an edgepath is the sum of lengths of edges in the edgepath. Naturally the length of a constant edgepath should be zero. For an edgepath $\gamma,|\gamma|$ denotes the length of the edgepath.

2.1.4. Examples. Some examples of edgepaths for $2 / 5$-tangle are given in figure 5. In (1), three basic edgepaths $\langle 1\rangle-\langle 1 / 2\rangle-\langle 2 / 5\rangle,\langle 0\rangle-\langle 1 / 2\rangle-\langle 2 / 5\rangle$ and $\langle 0\rangle-\langle 1 / 3\rangle-\langle 2 / 5\rangle$ are drawn. The first and third ones are monotonically increasing and decreasing, while the second one is neither. In (2), the edgepath $\langle 1\rangle-\langle 1 / 2\rangle-\langle 1 / 3\rangle-\langle 2 / 5\rangle$ is not minimal and is not allowed, since $\langle 1 / 2\rangle-\langle 1 / 3\rangle-\langle 2 / 5\rangle$ consists of two edges of a common triangle. In (3), a type I edgepath $\left(\frac{1}{2}\langle 0\rangle+\frac{1}{2}\langle 1 / 3\rangle\right)-\langle 1 / 3\rangle-\langle 2 / 5\rangle$ and a type III edgepath $\langle 1 / 0\rangle-\langle 1\rangle-\langle 1 / 2\rangle-\langle 2 / 5\rangle$ are drawn. A thick dot on the horizontal edge $\langle 2 / 5\rangle-\langle 2 / 5\rangle^{\circ}$ in this figure is a constant edgepath, which is a rational point on the horizontal edge. In (4), type II edgepaths $\langle 2\rangle-\langle 1\rangle-\langle 1 / 2\rangle-\langle 2 / 5\rangle$, $\langle 0\rangle-\langle 1\rangle-\langle 1 / 2\rangle-\langle 2 / 5\rangle,\langle 1\rangle-\langle 0\rangle-\langle 1 / 3\rangle-\langle 2 / 5\rangle$ and $\langle-1\rangle-\langle 0\rangle-\langle 1 / 3\rangle-\langle 2 / 5\rangle$ are depicted. Note that the second one is also an example of a non-minimal edgepath, since the edgepath is not minimal at the vertex $\langle 1\rangle$.

2.1.5. Edgepath systems. By an edgepath system, we mean a collection of $N$ edgepaths. Recall that $N$ denotes the number of tangles of a Montesinos knot. We will use symbol $\Gamma$ for edgepath systems.

We call the following conditions gluing consistency: the $u$-coordinates of the left endpoints of the edgepaths must coincide with each other, and the sum of $v$-coordinates of the left endpoints must be 0 .

As we will see, this condition must be satisfied by an edgepath system which corresponds to a properly embedded surface in the exterior of a Montesinos knot.

Edgepaths in an edgepath system satisfying the gluing consistency have the common $u$-coordinate of the left endpoints. Therefore, similar to 


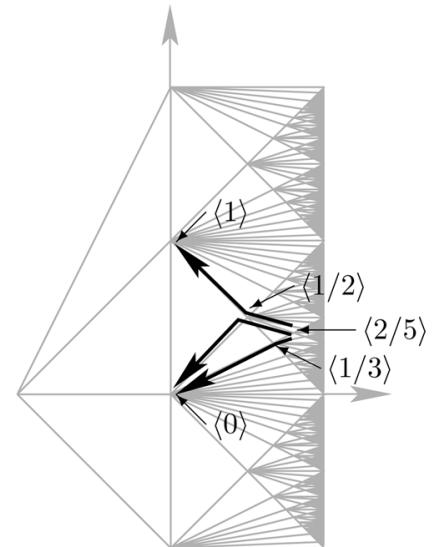

(1)

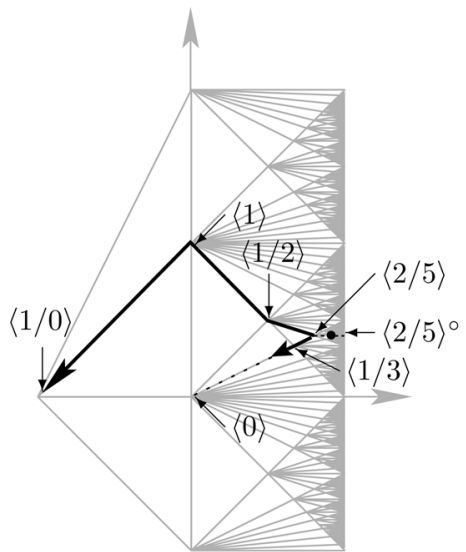

(3)

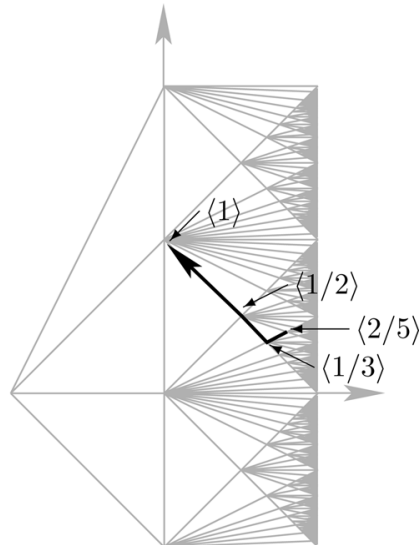

(2)

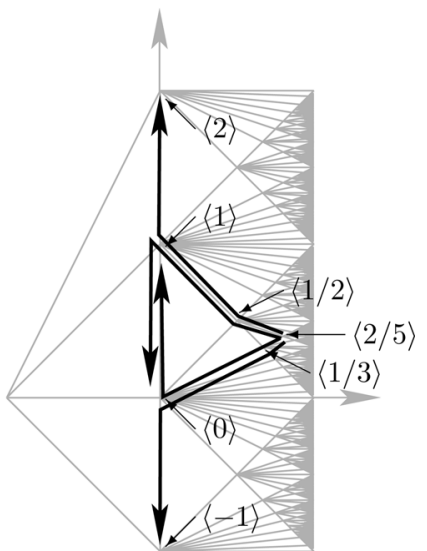

(4)

Figure 5: Examples of edgepaths for 2/5-tangle.

edgepaths, edgepath systems satisfying the gluing consistency are also classified by the common $u$-coordinates. That is, such an edgepath system is said to be of type I, type II or type III according to which of $u>0, u=0$ or $u<0$ the common $u$-coordinate satisfies.

In general, we will only consider edgepath systems with gluing consistency, but we sometimes make an edgepath system which may not satisfy the gluing consistency. We call the edgepath system a formal edgepath system.

For example, we will consider an edgepath system all of whose edgepaths are basic edgepaths, which we call a basic edgepath system. Such a basic edgepath system is formal. We usually use symbol $\Lambda$ for basic edgepath systems. 
2.1.6. Diagram and curve systems. We here observe a relationship between vertices and edges in $\mathcal{D}$ and curve systems on a fourth-punctured sphere. This enables us to describe a connection of edgepath systems and embedded surfaces in Montesinos knot exteriors. Such a connection will be explained in the next subsubsection.

As mentioned before, segments of slope $p / q$ on a pillowcase form two arcs. We call the arcs (a pair of) p/q-arcs. For instance, figure 6 (left) depicts $1 / 2$-arcs. For ease of drawing figures, we regard $\mathbb{R}^{2} \cup\{\infty\}$ as a sphere and draw the four punctures and $p / q$-arcs on the plane. See $1 / 2$-arcs in figure 6 (right). Some other examples of $p / q$-arcs are given in figure 7 . We define that the set of $m$ pairs of $p / q$-arcs correspond to the vertex $\langle p / q\rangle$ on $\mathcal{D}$. Please note that such multiple pairs can be drawn on the fourth-punctured sphere disjointly.

Next, consider the integers $p, q, r$ and $s$ satisfying $|p s-q r|=1$. Then a combination of $m$ pairs of $p / q$-arcs and $m-k$ pairs of $r / s$-arcs is set to correspond to a rational point $\frac{k}{m}\langle p / q\rangle+\frac{m-k}{m}\langle r / s\rangle$. Also note that such a pair of $p / q$-arcs and a pair of $r / s$-arcs can be disjointly drawn on a fourthpunctured sphere. A disjoint union of such pairs appears in figure 9.

Besides, a non-null-homotopic loop on a fourth-punctured sphere disjoint from $p / q$-arcs is topologically unique and is called a $p / q$-circle. We can think a disjoint union of $m$ pairs of $p / q$-arcs and $l$ copies of $p / q$-circles with $m \geq 1, l \geq 1$, as seen in figure 10 . This corresponds to a rational point $\frac{m}{m+l}\langle p / q\rangle+\frac{l}{m+l}\langle p / q\rangle^{\circ}$ of a horizontal edge.
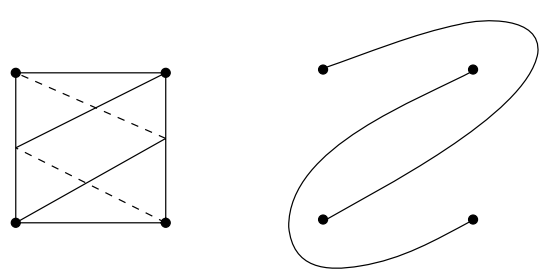

Figure 6: 1/2-arcs on a flattened sphere and on a plane.

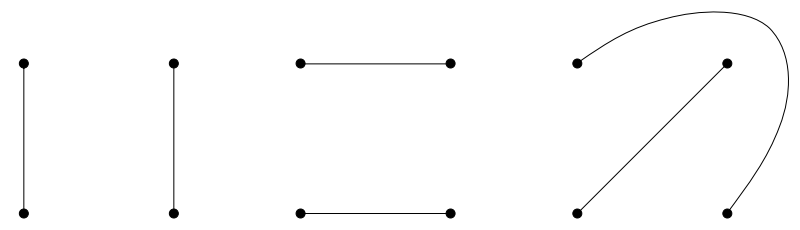

Figure 7: 1/0-arcs, 0-arcs, 1-arcs. 
2.1.7. Edgepath systems and corresponding surfaces. Consider the same setting as in the explanation of the outline of Hatcher-Oertel's machinery at the beginning of this subsection. Then a subsurface $F_{i}$ lies in one of the $N$ 3-balls. Now, we assume that the 3-ball is actually a unit ball with the center $O$. We think about the intersection between $F_{i}$ and a sphere with the center $O$ and radius $\rho>0$. As we vary $\rho$ within $0<\rho \leq 1$, the intersection changes. The subsurface $F_{i}$ is expressed by the sequence of such intersections between $F_{i}$ and concentric spheres.

By virtue of Proposition 1.1 in [5], the subsurface is deformed into a good position in Morse theoretical sense. This enables us to regard the intersection as a curve system, on any non-critical level $\rho$. The topological type of the intersection changes only at critical levels, and so, a subsurface can be represented by a sequence of curve systems and hence by an edgepath.

In the following, we observe parts of the surfaces represented by each edge in the edgepath system.

A non-constant edgepath corresponds to a saddle subsurface. An example of a saddle subsurface is expressed as a sequence of intersections with concentric spheres as illustrated in figure 8. In this figure, the intersection is empty for $\rho<1 / 4,1 / 2$-arcs for $1 / 4 \leq \rho<1 / 2$, a disk bounded by $1 / 2$-arcs and 0 -arcs for $\rho=1 / 2,0$-arcs for $1 / 2<\rho<3 / 4$, a disk bounded by 0 -arcs and $1 / 0$-arcs for $\rho=3 / 4$ and $1 / 0$-arcs for $3 / 4<\rho \leq 1$. This is a subsurface corresponding to an edgepath $\langle 1 / 0\rangle-\langle 0\rangle-\langle 1 / 2\rangle$. A disk bounded by two pairs of arcs is called a saddle. A pair of $p / q$-arcs and a pair of $r / s$-arcs can be disjointly drawn and give a saddle only if $|p s-q r|=1$. This is the reason why we think about an edgepath which consists of edges of the form $\langle p / q\rangle-\langle r / s\rangle$ satisfying $|p s-q r|=1$.

Note that, four punctures on each concentric sphere with radius $1 / 4<$ $\rho \leq 1$ and $1 / 2$-arcs on the sphere with radius $\rho=1 / 4$ form a $1 / 2$-tangle in

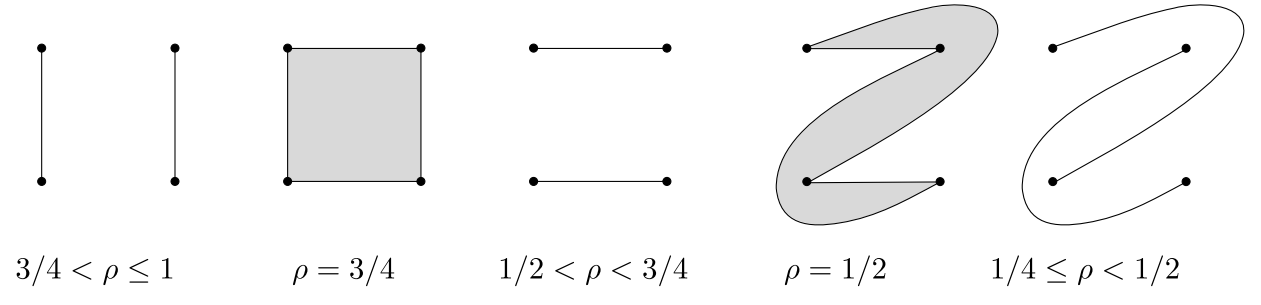

Figure 8: The intersection between a concentric sphere of radius $\rho$ and a subsurface expressed by an edgepath $\langle 1 / 0\rangle-\langle 0\rangle-\langle 1 / 2\rangle$. 
the 3-ball. Thus, the boundary of the subsurface lies on the boundary of the exterior of $1 / 2$-tangle.

The previous example of a subsurface is "one-sheeted." In general, a subsurface can have "multiple sheets." If a small meridian circle of a Montesinos knot $K$ meets an essential surface in $m$ points, then the surface is said to be $m$-sheeted, or the number of sheets of the surface is $m$ at the point on the knot. After isotoping the surface by keeping it properly embedded, the number of sheets is constant everywhere on the knot. Then, the number of sheets is defined for a surface. For an $m$-sheeted subsurface $F$, the intersection between $F$ and a concentric sphere is in general a curve system including exactly $m$ pairs of arcs.

Figure 9 illustrates an example of a saddle subsurface with multiple sheets. In this figure, there are three sheets, and only one saddle appears. The left figure is the union of one pair of $1 / 0$-arcs and two pairs of 0 -arcs, which is denoted by $\frac{1}{3}\langle 1 / 0\rangle+\frac{2}{3}\langle 0\rangle$. Then, this subsurface corresponds to a partial edge $\left(\frac{1}{3}\langle 1 / 0\rangle+\frac{2}{3}\langle 0\rangle\right)-\langle 0\rangle$. An edgepath including a partial edge must be multiple-sheeted.

Note that, since $\langle p / q\rangle$ corresponds also to a disjoint union of $m$ pairs of $p / q$-arcs for arbitrary positive integer $m$, an $m$-sheeted parallel subsurface also corresponds to an edgepath without a partial edge.

A subsurface corresponding to a constant edgepath is illustrated in figure 10. The intersection is empty for $\rho<1 / 3$, a disk bounded by a 0 -circle for $\rho=1 / 3$, a 0 -circle for $1 / 3<\rho<2 / 3$, and the union of a 0 -circle and 0 -arcs for $2 / 3 \leq \rho \leq 1$. Such a subsurface is called a cap subsurface. Note that the boundary of this subsurface lies on the boundary of the exterior of 0 -tangle in the 3-ball. The intersection between the subsurface and a concentric sphere of radius $2 / 3 \leq \rho \leq 1$ is expressed by a rational point $\frac{1}{2}\langle 0\rangle+\frac{1}{2}\langle 0\rangle^{\circ}$ and the subsurface is also denoted by $\frac{1}{2}\langle 0\rangle+\frac{1}{2}\langle 0\rangle^{\circ}$, which means a constant edgepath.

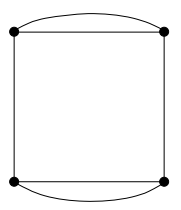

$2 / 3<\rho \leq 1$

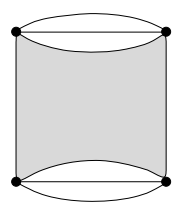

$\rho=2 / 3$

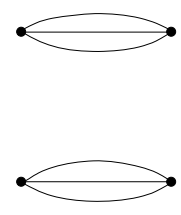

$1 / 3 \leq \rho<2 / 3$

Figure 9: The intersection between a concentric sphere of radius $\rho$ and a subsurface expressed by an edgepath $\left(\frac{1}{3}\langle 1 / 0\rangle+\frac{2}{3}\langle 0\rangle\right)-\langle 0\rangle$. 


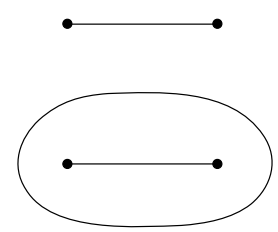

$2 / 3 \leq \rho \leq 1$

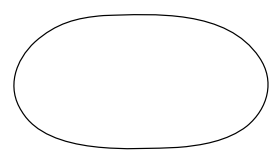

$1 / 3<\rho<2 / 3$

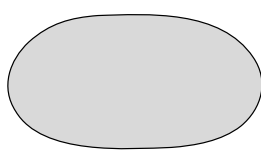

$\rho=1 / 3$

Figure 10: The intersections between a concentric sphere of radius $\rho$ and a subsurface expressed by an edgepath $\left(\frac{1}{2}\langle 0\rangle+\frac{1}{2}\langle 0\rangle^{\circ}\right)$.

For a saddle subsurface or a cap subsurface, the boundary of the subsurface lies on the boundary of the exterior of the corresponding rational tangle. Therefore, after the subsurfaces are glued to each other, the boundary of the obtained surface lies on the boundary of the exterior of a Montesinos knot. In short, the surface is confirmed to be properly embedded.

2.1.8. Twists and boundary slopes. In order to calculate the boundary slope of an essential surface, we calculate the total number of twists, or in short twist. As well as the boundary slope, the twist also measures how many times the boundary of a surface runs around a knot in the counterclockwise direction of the meridian, while the boundary runs once along the knot. The twist of a surface is obtained by first calculating the twist for each subsurface and then summing up the twists. The twist of a subsurface is calculated as the sum of twists for each saddle in the subsurface. For a saddle corresponding to an $\infty$-edge like $\langle 1 / 0\rangle-\langle z\rangle$, the twists appear on a saddle at four punctures and cancel out each other as in figure 11 (left). Besides, for a saddle corresponding to a non- $\infty$-edge $\langle p / q\rangle-\langle r / s\rangle$, the twist is 2 or -2 as seen in figure 11 (right). The sign of the twist is determined by whether the edge is decreasing or increasing.
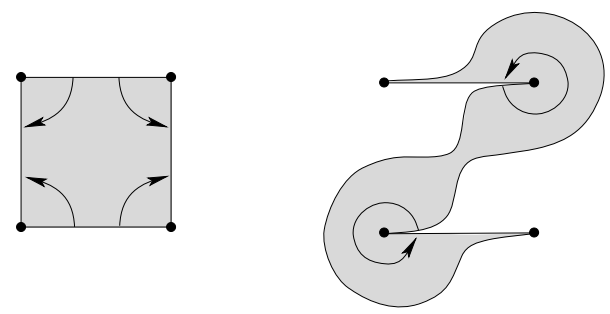

Figure 11: Saddles for $\langle 1 / 0\rangle-\langle 0\rangle$ and $\langle 0\rangle-\langle 1 / 2\rangle$. 
Next, for saddles of a subsurface with multiple sheets as in figure 9, a single saddle of an $m$-sheeted subsurface corresponding to a non- $\infty$-edge $\left(\frac{k}{m}\langle p / q\rangle+\frac{m-k}{m}\langle r / s\rangle\right)-\left(\frac{k+1}{m}\langle p / q\rangle+\frac{m-k-1}{m}\langle r / s\rangle\right)$ has the twist $\pm 2 / m$, and the twist for $\left(\frac{k}{m}\langle p / q\rangle+\frac{m-k}{m}\langle r / s\rangle\right)-\langle r / s\rangle$ is $\pm 2 k / m$. For a cap subsurface as in figure 10 , since no saddles appear, the twist is 0 .

Hence, the procedure to calculate the twist is summarized as follows. For each non- $\infty$-edge $e$ in an edgepath, we assign $-2 \sigma(e)|e|$ to the edge $e$. Then the twist of an edgepath is the sum of such assigned values for edges in the edgepath, where a constant edgepath has twist 0 . The twist of an edgepath system is the sum of twists of all edgepaths in the edgepath system. The twist is a substitution of the boundary slope and fits well with the algorithm. We use the symbol $\tau$ for the twist, and $\tau(e), \tau(\gamma), \tau(\Gamma)$ and $\tau(F)$ denote the twist of an edge $e$, an edgepath $\gamma$, an edgepath system $\Gamma$ and a surface $F$. Since the boundary slope is defined so that the boundary slope of a Seifert surface $F_{\text {Seifert }}$ is 0 , the boundary slope of an essential surface $F$ is calculated as the difference in the twist of the surface and that of a Seifert surface, that is, $\tau(F)-\tau\left(F_{\text {Seifert }}\right)$.

2.1.9. Remarks. We here collect some remarks about the previous subsubsections.

- Since each rational tangle $R_{i}$ in a Montesinos knot in this paper is nonintegral, the starting point of an edgepath must be a vertex $\langle p / q\rangle$ or a rational point on a horizontal edge $\langle p / q\rangle-\langle p / q\rangle^{\circ}$ for a non-integral fraction $p / q$. On the other hand, figures 9 and 10 correspond to edgepaths with the starting point on $\langle 0\rangle-\langle 0\rangle^{\circ}$. Hence, these figures are not appropriate in some sense. These figures are used just for ease of drawing and explanation.

- Here we illustrates three-dimensional figures of subsurfaces corresponding to a non-constant edgepath and a constant edgepath (see figure 12). Note that these subsurfaces lie in 3-balls and top boundaries of these subsurfaces lie on the boundary spheres of the 3-balls, which are not drawn in this figure. Besides, the "cap" of the cap subsurface in figure $12(\mathrm{~b})$ is somewhat deformed from the subsurface illustrated in figure 10 .

- Strictly speaking, there are some irregular essential surfaces: a Conway sphere and an augmented surface. Although, we can ignore these surfaces since their boundary slopes are $\infty$ or the same as that of some regular essential surface. See [5] for details. 

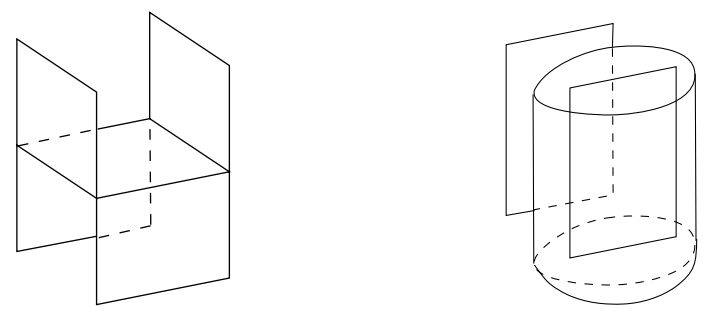

Figure 12: Examples of subsurfaces: (a) a saddle subsurface corresponding to $\langle\infty\rangle-\langle 0\rangle$; (b) a cap subsurface corresponding to $\frac{1}{2}\langle 0\rangle+\frac{1}{2}\langle 0\rangle^{\circ}$.

\subsection{Crossing number of Montesinos knot}

Here, we will explain the result given in [9] on the calculation of the crossing number for a Montesinos knot.

2.2.1. Alternating tangle diagram. Assume that $R$ is a non-integral fraction. There is the unique continued fraction of $R$ as

$$
R=z_{1}+\frac{1}{z_{2}+\frac{1}{\ddots \frac{1}{z_{k-1}+\frac{1}{z_{k}}}}}
$$

with $k \geq 2$ where the integers $z_{1}, z_{2}, \ldots, z_{k}$ satisfy

$$
\begin{array}{cc}
z_{1} \geq 0, z_{j} \geq 1(1<j<k), z_{k} \geq 2 & \text { if } R>0 \\
z_{1} \leq 0, z_{j} \leq-1(1<j<k), z_{k} \leq-2 & \text { if } R<0 .
\end{array}
$$

Let $\left(z_{1}, z_{2}, \ldots, z_{k}\right)$ denote this continued fraction. For instance, a continued fraction expansion of $47 / 36$ is

$$
\begin{aligned}
\frac{47}{36} & =1+\frac{11}{36}=1+\frac{1}{\frac{36}{11}}=1+\frac{1}{3+\frac{3}{11}}=1+\frac{1}{3+\frac{1}{\frac{11}{3}}} \\
& =\cdots=1+\frac{1}{3+\frac{1}{3+\frac{1}{1+\frac{1}{2}}}}=(1,3,3,1,2) .
\end{aligned}
$$

This continued fraction gives a diagram of a rational tangle $R$, which is roughly a combination of vertical and horizontal half-twists. See figure 13 for an example. If we connect two pairs of adjacent ends of the diagram, because of the condition on $z_{j} \mathrm{~s}$, we obtain an alternating diagram of a two-bridge link. Thus, we call a diagram like this of a rational tangle an alternating 


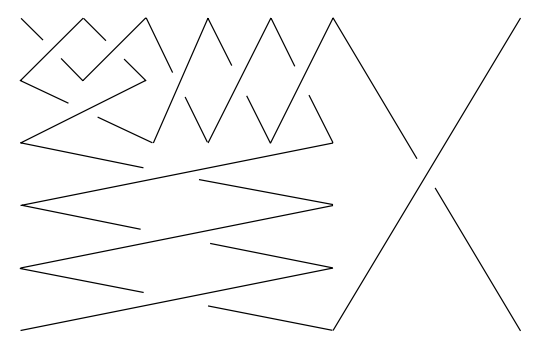

Figure 13: The alternating tangle diagram of the tangle $(1,3,3,1,2)=$ $47 / 36$.

tangle diagram of a rational tangle $R$, which is denoted by $D(R)$. Note that the number of crossings of the diagram is $\sum_{l=1}^{k}\left|z_{l}\right|$.

2.2.2. Reduced Montesinos diagram. In [9], Lickorish and Thistlethwaite determine the crossing number of the Montesinos knots. Though, we must perform a technical operation which restricts the form of a diagram of a Montesinos knot.

Let $K$ be a Montesinos knot $K=K\left(R_{1}, R_{2}, \ldots, R_{N}\right)$ with $N \geq 3$, where each $R_{j}$ is a non-integral fraction. For $\left(R_{1}, R_{2}, \ldots, R_{N}\right)$, let $D\left(R_{1}, R_{2}, \ldots, R_{N}\right)$ denote the diagram of $K$ obtained by combining alternating tangle diagrams $D\left(R_{1}\right), D\left(R_{2}\right), \ldots, D\left(R_{N}\right)$ in a natural way.

Now, for any tuple of integers $\left(i_{1}, i_{2}, \ldots, i_{N}\right)$ with $i_{1}+i_{2}+\cdots+i_{N}=0$, the Montesinos knot $K^{\prime}=K\left(R_{1}+i_{1}, R_{2}+i_{2}, \ldots, R_{N}+i_{N}\right)$ is isotopic to $K$, since addition of such integers corresponds to rotations of the tangles. Thus, for instance, if $\left\{R_{j}\right\}$ includes both positive and negative fractions and $R_{a}>1$ for some $a$, by subtracting 1 from $R_{a}$ and adding 1 to some negative $R_{b}$, we can reduce the integral part $\left[R_{a}\right]$ of $R_{a}$. Through repeating such operations, we finally have a tuple $\left(R_{1}^{\prime}, R_{2}^{\prime}, \ldots, R_{N}^{\prime}\right)$ satisfying

(A) all $R_{j}^{\prime}$ 's have the same sign

or

(B) $\left\{R_{j}^{\prime}\right\}$ includes both positive and negative fractions, and $\left|R_{j}^{\prime}\right|<1$ holds for all $j$.

For $\left(R_{1}, R_{2}, \ldots, R_{N}\right)$ satisfying $(\mathrm{A})$ or $(\mathrm{B})$, we think about the diagram $D=D\left(R_{1}, R_{2}, \ldots, R_{N}\right)$. If $\left\{R_{j}\right\}$ have the common sign, then the diagram satisfies condition (i) in [9], that is, the diagram is alternating. If $\left|R_{j}\right|<1$ holds for each $j$, then each alternating tangle diagram $D\left(R_{j}\right)$ in the diagram $D$ satisfies a technical condition in the condition (ii). Therefore, $D$ satisfies the condition (i) or (ii) in [9] to be a reduced Montesinos diagram. 
With this diagram, the crossing number of a Montesinos knot $K$ is given by the following.

Theorem 10 of [9]. If a link $L$ admits an $n$-crossing, reduced Montesinos diagram, then $L$ cannot be projected with fewer than $n$ crossings.

In short, in order to calculate the crossing number of a given Montesinos knot $K$, we have only to prepare a reduced Montesinos diagram and count its crossings.

Thus, by putting them all together, we have the following.

Proposition 2.1. For a Montesinos knot $K=K\left(R_{1}, R_{2}, \ldots, R_{N}\right)$, there is a tuple $\left(R_{1}^{\prime}, R_{2}^{\prime}, \ldots, R_{N}^{\prime}\right)$ satisfying either $(A)$ or $(B)$, such that the knot $K$ is isotopic to $K^{\prime}=K\left(R_{1}^{\prime}, R_{2}^{\prime}, \ldots, R_{N}^{\prime}\right)$ and has a diagram $D=D\left(R_{1}^{\prime}, R_{2}^{\prime}, \ldots\right.$, $\left.R_{N}^{\prime}\right)$. Furthermore, the diagram $D$ is a reduced Montesinos diagram defined in [9] and attains the crossing number of $K$.

\section{Proof of the main theorem}

The key of the proof is the relation between the number of crossings of the alternating diagram of a rational tangle and lengths of monotonic edgepaths for the rational tangle. Roughly, summing up these relations gives the main theorem, since the crossing number of a Montesinos knot is calculated as the sum of the numbers of crossings of the alternating diagrams of rational tangles by [9].

\subsection{Number of crossings and lengths of monotonic edgepaths}

We start with a single rational tangle.

Let $\operatorname{cr}(D)$ denote the number of crossings of a tangle diagram $D$. The number $\operatorname{cr}(D(R))$ of crossings of the alternating tangle diagram $D(R)$ of a rational tangle $R$ is related to the lengths of monotonic edgepaths for $R$ by the following lemma.

Let $\lambda_{\text {inc }}$ and $\lambda_{\text {dec }}$ be the monotonically increasing and decreasing basic edgepaths, which are determined uniquely for given $R$. By adding $\infty$-edges, we obtain the monotonically increasing and decreasing type III edgepaths $\gamma_{\text {III,inc }}$ and $\gamma_{\text {III,dec }}$ from the basic edgepaths. Note that, since $\infty$-edges do not contribute to the twist, we ignore $\infty$-edges when we judge a type III edgepath is monotonic or not. According to the sign of $R$, we can construct 
a monotonic type II edgepath with an ending vertex $\langle 0\rangle$, which is a monotonically increasing type II edgepath $\gamma_{\mathrm{II} \text {,inc }}$ if $R<0$ and a monotonically decreasing type II edgepath $\gamma_{\mathrm{II} \text {,dec }}$ if $R>0$.

Then, we obtain the following key lemma.

Lemma 3.1. Assume that $R$ is a non-integral fraction. Then,

$$
\begin{array}{ll}
\operatorname{cr}(D(R))=\left|\gamma_{\mathrm{III}, \text { inc }, \geq 0}\right|+\left|\gamma_{\mathrm{II}, \mathrm{dec}}\right| & \text { if } R>0, \\
\operatorname{cr}(D(R))=\left|\gamma_{\mathrm{II}, \text { inc }}\right|+\left|\gamma_{\mathrm{III}, \mathrm{dec}, \geq 0}\right| & \text { if } R<0 .
\end{array}
$$

Furthermore,

$$
\operatorname{cr}(D(R))=\left|\gamma_{\mathrm{III}, \mathrm{inc}, \geq 0}\right|+\left|\gamma_{\mathrm{III}, \mathrm{dec}, \geq 0}\right| \quad \text { if }|R|<1
$$

$\gamma_{*, \geq 0}$ denotes the part of $\gamma_{*}$ lying in $u \geq 0$.

Proof. Assume that $R$ is positive, and is represented by a continued fraction $\left(z_{1}, z_{2}, \ldots, z_{k}\right)$ satisfying $k \geq 2$ and the condition (2.1). This continued fraction corresponds to the alternating diagram $D(R)$ of the rational tangle $R$. We also prepare monotonic edgepaths $\gamma_{\mathrm{III} \text {,inc }}$ and $\gamma_{\mathrm{II}, \mathrm{dec}}$.

We divide these monotonic edgepaths by the use of triangles defined as follows. For integers $2 \leq l \leq k$ and $m \geq 1$, there exists a large triangle with vertices

$$
\left\langle\left(z_{1}, z_{2}, \ldots, z_{l-1}\right)\right\rangle,\left\langle\left(z_{1}, z_{2}, \ldots, z_{l-1}, 1\right)\right\rangle,\left\langle\left(z_{1}, z_{2}, \ldots, z_{l-1}, m\right)\right\rangle .
$$

See figure 15. Note here that an expression like $\left(z_{1}, z_{2}, \ldots, z_{a}\right)$ denotes a rational number $R$ corresponding to the continued fraction, and hence, $\left\langle\left(z_{1}, z_{2}, \ldots, z_{a}\right)\right\rangle$ denotes a vertex $\langle R\rangle$ of the diagram $\mathcal{D}$. Moreover, this triangle consists of some smaller triangles with vertices

$$
\left\langle\left(z_{1}, z_{2}, \ldots, z_{l-1}\right)\right\rangle,\left\langle\left(z_{1}, z_{2}, \ldots, z_{l-1}, j\right)\right\rangle,\left\langle\left(z_{1}, z_{2}, \ldots, z_{l-1}, j+1\right)\right\rangle
$$

for $1 \leq j \leq m-1$. The existence of such a triangle is explained roughly as follows.

An important fact here is that if $\left(z_{1}, z_{2}, \ldots, z_{l}\right)=p / q$ and $\left(z_{1}, z_{2}, \ldots\right.$, $\left.z_{l}, 1\right)=r / s$ with $|p s-q r|=1$, then $\left(z_{1}, z_{2}, \ldots, z_{l}, j\right)=\{(j-1) p+r\} /\{(j-$ $1) q+s\}$ holds for any $j \geq 1$. This is easily shown for $(0, j) \mathrm{s}$, and then for $\left(z_{l}, j\right) \mathrm{s},\left(0, z_{l}, j\right) \mathrm{s},\left(z_{l-1}, z_{l}, j\right) \mathrm{s}$ and so on, inductively. With the fact, the following features are easily confirmed. There are edges $\left\langle\left(z_{1}, z_{2}, \ldots, z_{l}\right)\right\rangle-$ $\left\langle\left(z_{1}, z_{2}, \ldots, z_{l}, j\right)\right\rangle$ and $\left\langle\left(z_{1}, z_{2}, \ldots, z_{l}, j\right)\right\rangle-\left\langle\left(z_{1}, z_{2}, \ldots, z_{l}, j+1\right)\right\rangle$. For the 
sequence of vertices $\left\langle\left(z_{1}, z_{2}, \ldots, z_{l}, 1\right)\right\rangle,\left\langle\left(z_{1}, z_{2}, \ldots, z_{l}, 2\right)\right\rangle, \ldots$, their $v$-coordinates are monotonic and their $u$-coordinates are monotonically increasing. For all edges of the form $\left\langle\left(z_{1}, z_{2}, \ldots, z_{l}, j\right)\right\rangle-\left\langle\left(z_{1}, z_{2}, \ldots, z_{l}, j+1\right)\right\rangle$, if we regard them as line segments, then the slopes of the segments coincide with each other.

This is because we have

$$
\begin{aligned}
\left\langle\left(z_{1}, z_{2}, \ldots, z_{l}, j\right)\right\rangle & =\left\langle\frac{(j-1) p+r}{(j-1) q+s}\right\rangle, \\
\left\langle\left(z_{1}, z_{2}, \ldots, z_{l}, j+1\right)\right\rangle & =\left\langle\frac{j p+r}{j q+s}\right\rangle,
\end{aligned}
$$

their $u v$-coordinates are

$$
\left(1-\frac{1}{(j-1) q+s}, \frac{(j-1) p+r}{(j-1) q+s}\right) \quad \text { and } \quad\left(1-\frac{1}{j q+s}, \frac{j p+r}{j q+s}\right)
$$

and the slope of the segment between them is found to be

$$
\frac{p s-q r}{q}
$$

which does not depend on $j$. Hence, the vertices $\left\langle\left(z_{1}, z_{2}, \ldots, z_{l}, j\right)\right\rangle$ 's for $j \geq 1$ lie on the same line. Thus, we have confirmed the existence of the triangle mentioned as before.

Now, for $l=1$, let $\gamma_{\text {inc }, l}=\langle\infty\rangle-\left\langle z_{1}+1\right\rangle, \gamma_{\text {dec }, l}=\langle 0\rangle-\langle 1\rangle-\cdots-\left\langle z_{1}\right\rangle$. We have $\left|\gamma_{\text {dec }, l}\right|=z_{1}$.

For $2 \leq l \leq k-1$, we focus on the triangle $T_{l}$ having the vertices

$$
\left\langle\left(z_{1}, z_{2}, \ldots, z_{l-1}\right)\right\rangle,\left\langle\left(z_{1}, z_{2}, \ldots, z_{l-1}, 1\right)\right\rangle,\left\langle\left(z_{1}, z_{2}, \ldots, z_{l-1}, z_{l}+1\right)\right\rangle .
$$

See figure 14 if $l=2$, figure 15 if $3 \leq l \leq k-1$. The triangle $T_{l}$ looks like either of the two large triangles in figure 15, depending on the parity of $l$. The large triangle $T_{l}$ includes two edgepaths

$$
\begin{aligned}
\gamma_{l, A}= & \left\langle\left(z_{1}, z_{2}, \ldots, z_{l-1}, 1\right)\right\rangle-\left\langle\left(z_{1}, z_{2}, \ldots, z_{l-1}, 2\right)\right\rangle \\
& -\cdots-\left\langle\left(z_{1}, z_{2}, \ldots, z_{l-1}, z_{l}-1\right)\right\rangle-\left\langle\left(z_{1}, z_{2}, \ldots, z_{l-1}, z_{l}\right)\right\rangle, \\
\gamma_{l, B 1}= & \left\langle\left(z_{1}, z_{2}, \ldots, z_{l-1}\right)\right\rangle-\left\langle\left(z_{1}, z_{2}, \ldots, z_{l-1}, z_{l}+1\right)\right\rangle .
\end{aligned}
$$

One of the two edgepaths is monotonically increasing, and the other is monotonically decreasing. Note that $\gamma_{l, A}$ can be a point when $z_{l}=1$, and such an edgepath can be regarded as both increasing and decreasing. Let $\gamma_{\text {inc, } l}$ 


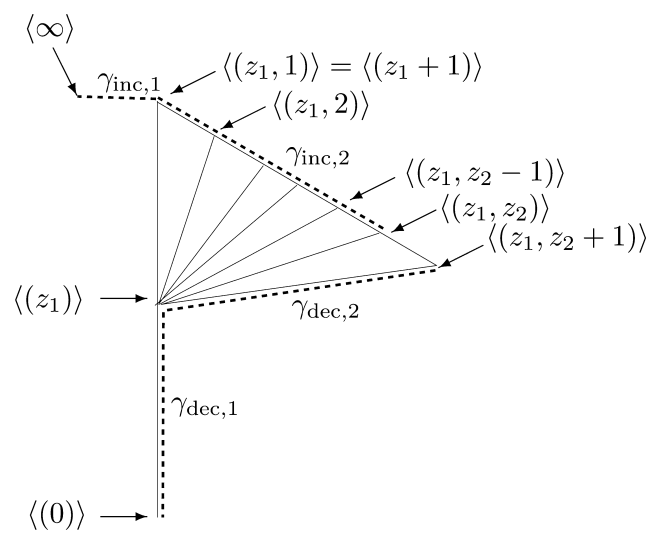

Figure 14: $\gamma_{\text {inc }, l}$ and $\gamma_{\mathrm{dec}, l}$ for $l=1$ and $l=2$.
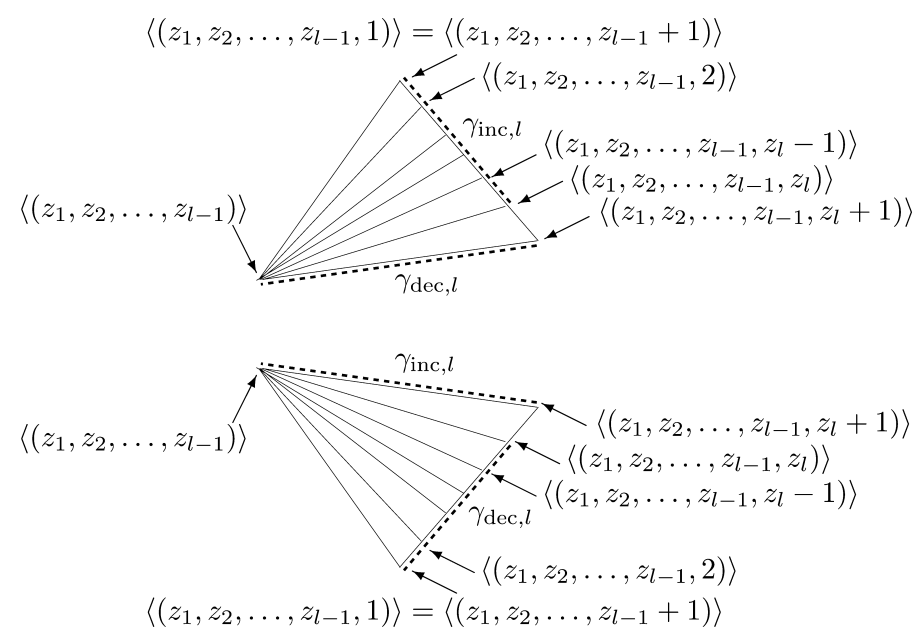

Figure 15: $\gamma_{\text {inc, } l}$ and $\gamma_{\mathrm{dec}, l}$ in a large triangle $T_{l}$ for $3 \leq l \leq k-1$.

and $\gamma_{\mathrm{dec}, l}$ denote the increasing and the decreasing ones. The important fact here is that $\left|\gamma_{\mathrm{inc}, l}\right|+\left|\gamma_{\mathrm{dec}, l}\right|=z_{l}-1+1=z_{l}$.

For $l=k$, we define $\left\langle\left(z_{1}, z_{2}, \ldots, z_{l-1}\right)\right\rangle\left\langle\left(z_{1}, z_{2}, \ldots, z_{l-1}, 1\right)\right\rangle\left\langle\left(z_{1}, z_{2}, \ldots\right.\right.$, $\left.\left.z_{l-1}, z_{l}\right)\right\rangle$ as the triangle $T_{l}$. We use

$$
\gamma_{l, B 2}=\left\langle\left(z_{1}, z_{2}, \ldots, z_{l-1}\right)\right\rangle-\left\langle\left(z_{1}, z_{2}, \ldots, z_{l-1}, z_{l}\right)\right\rangle
$$

instead of $\gamma_{l, B 1}$. Though, the situation is similar to the previous case, and hence, the identity $\left|\gamma_{\text {inc }, l}\right|+\left|\gamma_{\text {dec }, l}\right|=z_{l}$ still holds (see figure 16). 


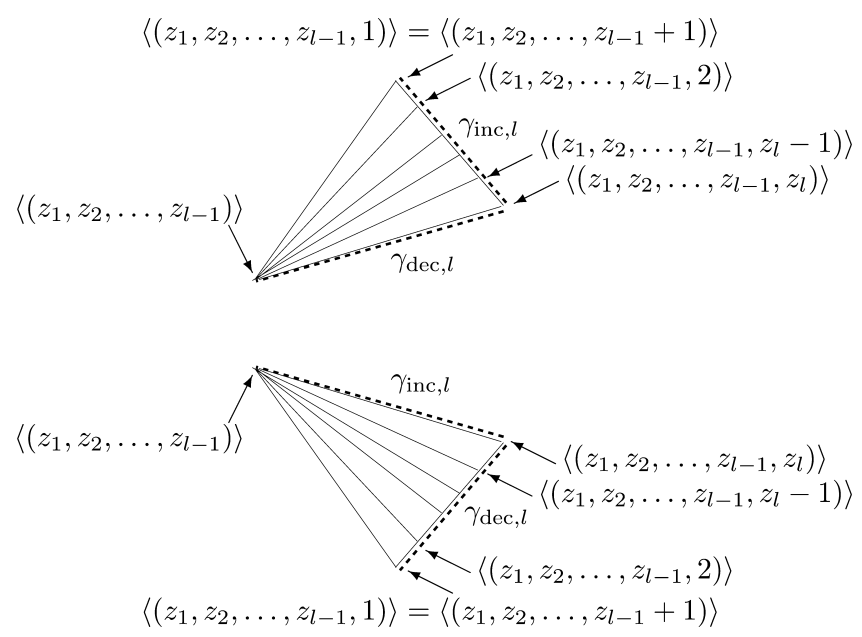

Figure 16: $\gamma_{\text {inc }, l}$ and $\gamma_{\text {dec }, l}$ in a large triangle $T_{l}$ for $l=k$.

By paying attention to the fact that $\left(z_{1}, z_{2}, \ldots, z_{l}+1\right)=\left(z_{1}, z_{2}, \ldots, z_{l}, 1\right)$, we can find that $\gamma_{\mathrm{III} \text {,inc }}$ is obtained by combining $\gamma_{\mathrm{inc}, l}(1 \leq l \leq k)$, and $\gamma_{\mathrm{II} \text {,dec }}$ by combining $\gamma_{\mathrm{dec}, l}(1 \leq l \leq k)$. Furthermore, we have

$$
\left|\gamma_{\mathrm{III}, \text { inc }, \geq 0}\right|+\left|\gamma_{\mathrm{II}, \mathrm{dec}}\right|=\sum_{l=2}^{k}\left(\left|\gamma_{\mathrm{inc}, l}\right|+\left|\gamma_{\mathrm{dec}, l}\right|\right)+\left|\gamma_{\mathrm{dec}, 1}\right|=\sum_{l=1}^{k} z_{l}=\operatorname{cr}(D(R)) .
$$

The argument is similar for negative $R$.

If $|R|<1$, then $z_{1}$ is 0 . Hence, we have

$$
\left|\gamma_{\mathrm{III}, \text { inc }, \geq 0}\right|+\left|\gamma_{\mathrm{III}, \mathrm{dec}, \geq 0}\right|=\operatorname{cr}(D(R))
$$

For instance, in figure 17, $\gamma_{\mathrm{III}, \text { inc }}$ and $\gamma_{\mathrm{II}, \mathrm{dec}}$ are depicted for $47 / 36=$ $(1,3,3,1,2)$. In the figure, for making it clear, the horizontal coordinates of the vertices are somewhat altered from correct values, though, it does not matter in the argument. Note that $\left|\gamma_{\mathrm{III}, \text { inc }, \geq 0}\right|+\left|\gamma_{\mathrm{II}, \mathrm{dec}}\right|=4+6=10$ coincides with $\operatorname{cr}(D(47 / 36))=1+3+3+1+2=10$.

By the argument in the above proof, we obtained the following about the possible edgepaths.

Proposition 3.2. The twists of monotonic basic edgepath systems give the upper and lower bounds for twists of basic edgepath systems, of type I edgepath systems and of type III edgepath systems. 


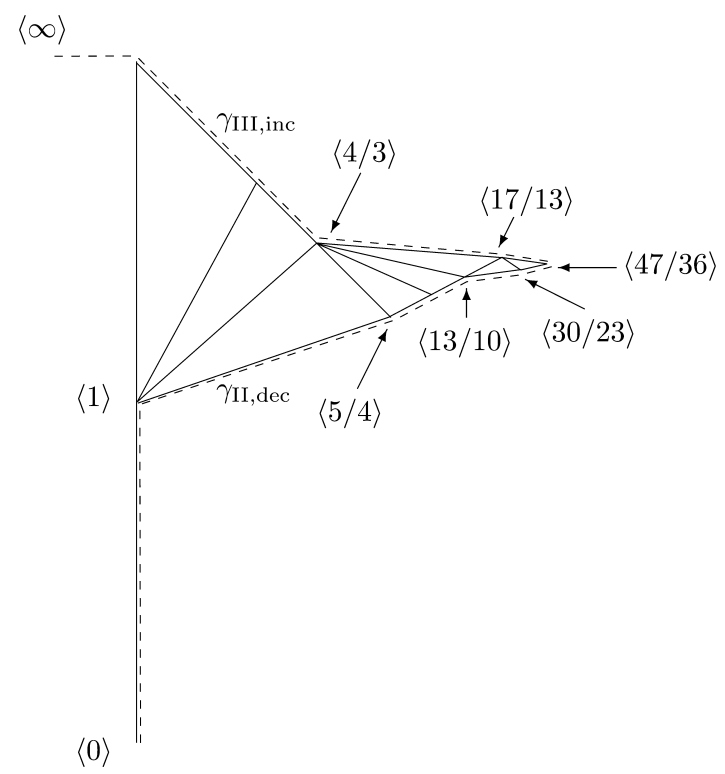

Figure 17: $\gamma_{\mathrm{III} \text {,inc }}$ and $\gamma_{\mathrm{II}, \mathrm{dec}}$, for $R=47 / 36$.

Proof. Let $\Lambda_{\text {inc }}=\left(\lambda_{\text {inc }, 1}, \ldots, \lambda_{\text {inc }, N}\right)$ and $\Lambda_{\text {dec }}=\left(\lambda_{\text {dec }, 1}, \ldots, \lambda_{\text {dec }, N}\right)$ denote the unique monotonically increasing basic edgepath system and the unique monotonically decreasing basic edgepath system. $\Gamma=\left(\gamma_{1}, \ldots, \gamma_{N}\right)$ denotes a basic edgepath system, a type I edgepath system or a type III edgepath system. If $\tau\left(\lambda_{\text {inc }, i}\right) \leq \tau\left(\gamma_{i}\right) \leq \tau\left(\lambda_{\text {dec }, i}\right)$ holds for each $i$, then we immediately have $\sum_{i=1}^{N} \tau\left(\lambda_{\text {inc }, i}\right) \leq \sum_{i=1}^{N} \tau\left(\gamma_{i}\right) \leq \sum_{i=1}^{N} \tau\left(\lambda_{\text {dec }, i}\right)$, or equivalently, $\tau\left(\Lambda_{\text {inc }}\right) \leq \tau(\Gamma) \leq \tau\left(\Lambda_{\text {dec }}\right)$. Hence, it suffices to show that $\tau\left(\lambda_{\text {inc }}\right) \leq \tau(\gamma) \leq$ $\tau\left(\lambda_{\text {dec }}\right)$ holds for each edgepath $\gamma=\gamma_{i}$.

For a fixed starting point $\langle R\rangle=\langle p / q\rangle$, all non-constant edgepaths lie in the region $X$ bounded by the monotonically increasing basic edgepath $\lambda_{\text {inc }}$, the monotonically decreasing basic edgepath $\lambda_{\text {dec }}$ and exactly one vertical edge as illustrated in figure 17. In this figure, $X$ is the union of eight triangles. As seen from the figure, roughly, the region $X$ consists of a sequence of triangles. Hence, no vertices of $\gamma$ other than a rational endpoint lie in the interior of $X$. All increasing (complete) edges in the region end at a vertex on $\lambda_{\text {inc }}$. For each intermediate vertex on $\lambda_{\text {inc }}$, there are one or more increasing edges ending at the vertex. A single edgepath contains at most one of them, and just one of them is always included in $\lambda_{\text {inc }}$. This implies that the number of increasing edges in an edgepath is less than or equal to that of the monotonically increasing basic edgepath. At the same 
time, the number of decreasing edges in an edgepath is more than or equal to that of the monotonically increasing basic edgepath, which is zero. Recall that the twist can be calculated as twice as the total length of the decreasing non- $\infty$-edges minus the total length of increasing non- $\infty$-edges. Hence, we have $\tau\left(\lambda_{\text {inc }}\right) \leq \tau(\gamma)$. The argument is similar for the monotonically decreasing basic edgepath.

\subsection{Crossing number and diameter}

We sum up identities in Lemma 3.1 to all rational tangles of a Montesinos knot, and obtain the relation between the diameter and the crossing number.

We separate the argument according to which of the conditions (A) and (B) is satisfied.

3.2.1. Alternating case. If a tuple $\left(R_{1}, R_{2}, \ldots, R_{N}\right)$ satisfies the condition (A), then the Montesinos knot $K\left(R_{1}, R_{2}, \ldots, R_{N}\right)$ is alternating. Conversely, by the following well-known fact, together with the another well-known fact that any Montesinos knot without 1/0-tangles is prime, an alternating Montesinos knot is isotopic to a Montesinos knot with a tuple satisfying not (B) but (A).

Proposition 3.3 (e.g., [8]). If a link L has a connected, irreducible, alternating diagram with $n$ crossings, then the number of crossings of any diagram of the link $L$ is $n$ or greater. Moreover, the number of crossings of any prime, non-alternating diagram is greater than $n$.

In this case, the situation is rather simple, and we have the following lemma.

Lemma 3.4. For a tuple $\left(R_{1}, R_{2}, \ldots, R_{N}\right)$, assume that all $R_{j}$ s have the common sign. Then, for the Montesinos knot $K=K\left(R_{1}, R_{2}, \ldots, R_{N}\right)$, the following identity holds.

$$
2 \operatorname{cr}(K)=\operatorname{Diam}(K)
$$

Proof. Assume here that all $R_{j}$ s are positive. As mentioned before, by Theorem 10 of $[9], \operatorname{cr}(K)=\sum_{j=1}^{N} \operatorname{cr}\left(D\left(R_{j}\right)\right)$. Let $\Gamma_{\text {III,inc }}$ and $\Gamma_{\text {II,dec }}$ denote the monotonically increasing type III edgepath system and the monotonically decreasing type II edgepath system whose edgepaths are constructed in the same manner as in Subsection 3.1. With respect to these, by Lemma 3.1, we have $\operatorname{cr}\left(D\left(R_{j}\right)\right)=\left|\gamma_{\mathrm{III}, \text { inc }, j, \geq 0}\right|+\left|\gamma_{\mathrm{II}, \mathrm{dec}, j}\right|$ for any $j$. One complete 
edge contributes \pm 2 to the twist. The twists of two edgepath systems $\Gamma_{\text {III,inc }}$ and $\Gamma_{\text {II,dec }}$ have opposite signs. Combining these facts, we have:

$$
\begin{aligned}
2 \operatorname{cr}(K) & =2 \sum_{j=1}^{N} \operatorname{cr}\left(D\left(R_{j}\right)\right)=2 \sum_{j=1}^{N}\left(\left|\gamma_{\mathrm{III}, \text { inc }, j, \geq 0}\right|+\left|\gamma_{\mathrm{II}, \mathrm{dec}, j}\right|\right) \\
& =\sum_{j=1}^{N} 2\left|\gamma_{\mathrm{III}, \text { inc }, j, \geq 0}\right|+\sum_{j=1}^{N} 2\left|\gamma_{\mathrm{II}, \mathrm{dec}, j}\right|=\left|\tau\left(\Gamma_{\mathrm{III}, \text { inc }}\right)\right|+\left|\tau\left(\Gamma_{\mathrm{II}, \mathrm{dec}}\right)\right| \\
& =\left|\tau\left(\Gamma_{\mathrm{III}, \text { inc }}\right)-\tau\left(\Gamma_{\mathrm{II}, \mathrm{dec}}\right)\right| .
\end{aligned}
$$

Now, both $\Gamma_{\text {III,inc }}$ and $\Gamma_{\text {II,dec }}$ are minimal. $\Gamma_{\text {III,inc gives an essential }}$ surface by Proposition 2.5 of [5] since the sum of $v$-coordinates at $u=0$ of the edgepaths in the edgepath system $\Gamma_{\text {III,inc }}$ is at least 3 . In addition, $\Gamma_{\text {II,dec }}$ corresponds to an essential surface by one of Proposition 2.4, 2.6, 2.7, 2.8 of [5] since it is monotonic.

For a type I or type III edgepath system $\Gamma$, we have $\tau\left(\Gamma_{\text {III,inc }}\right)=\tau\left(\Lambda_{\text {inc }}\right) \leq$ $\tau(\Gamma) \leq \tau\left(\Lambda_{\mathrm{dec}}\right) \leq \tau\left(\Gamma_{\mathrm{II}, \mathrm{dec}}\right)$ by Proposition 3.2. If $\Gamma=\left(\gamma_{1}, \gamma_{2}, \ldots, \gamma_{N}\right)$ is type II, there is another type II edgepath system $\Gamma^{\prime}=\left(\gamma_{1}^{\prime}, \gamma_{2}^{\prime}, \ldots, \gamma_{N}^{\prime}\right)$ each of whose edgepath $\gamma_{i}^{\prime}$ shares a basic edgepath with $\gamma_{i}$ and ends at $\langle 0\rangle$. Note that $\tau(\Gamma)=\tau\left(\Gamma^{\prime}\right)$ holds since the edgepath systems share a basic edgepath system and have the same total amount of signed length of vertical edges by the gluing consistency. By an argument similar to Proposition 3.2 and considering the effect of vertical edges on twist, we have $\tau\left(\Gamma_{\text {III,inc }}\right)=\tau\left(\Lambda_{\text {inc }}\right) \leq$ $\tau\left(\Gamma^{\prime}\right) \leq \tau\left(\Gamma_{\text {II,dec }}\right)$. Hence, $\tau\left(\Gamma_{\text {III,inc }}\right) \leq \tau(\Gamma) \leq \tau\left(\Gamma_{\text {II,dec }}\right)$ holds for type II edgepath systems, and hence for edgepath systems of any type.

In short, $\Gamma_{\text {III,inc }}$ and $\Gamma_{\text {II,dec }}$ are found to give the minimal and the maximal twists. Finally,

$$
2 \operatorname{cr}(K)=\left|\tau\left(\Gamma_{\text {III,inc }}\right)-\tau\left(\Gamma_{\text {II,dec }}\right)\right|=\operatorname{Diam}(K) .
$$

3.2.2. Non-alternating case. Note that Lemma 3.4 as is cannot be extended to the general Montesinos knots. For instance, $K=K(1 / 2,1 / 3$, $-1 / 3)=8_{20}$ has non-integral diameter $38 / 3$. If a tuple $\left(R_{1}, R_{2}, \ldots, R_{N}\right)$ satisfies the condition (B), then we have the following lemma.

Lemma 3.5. For a tuple $\left(R_{1}, R_{2}, \ldots, R_{N}\right)$, assume that $\left\{R_{j}\right\}$ includes both positive and negative fractions, and $\left|R_{j}\right|<1$ holds for any $j$. Then, for the 
Montesinos knot $K=K\left(R_{1}, R_{2}, \ldots, R_{N}\right)$, the following inequality holds.

$$
2 \operatorname{cr}(K) \geq \operatorname{Diam}(K)
$$

Proof. First, we prepare monotonic type III edgepath systems $\Gamma_{\text {III,inc }}$ and $\Gamma_{\text {III,dec. }}$ Similar to the argument in the proof of the previous lemma, we have

$$
2 \operatorname{cr}(K)=\left|\tau\left(\Gamma_{\text {III,inc }}\right)-\tau\left(\Gamma_{\text {III,dec }}\right)\right| \text {. }
$$

Note that the condition $\left|R_{j}\right|<1$ is required to apply Lemma 3.1.

We think about the range of twists. The twists of the monotonic type III edgepath systems are $\tau\left(\Gamma_{\text {III,inc }}\right)=\tau\left(\Lambda_{\text {inc }}\right)$ and $\tau\left(\Gamma_{\text {III,dec }}\right)=\tau\left(\Lambda_{\text {dec }}\right)$. Though, the surfaces corresponding to these edgepath systems may be inessential. We check that $\tau\left(\Gamma_{\text {III,inc }}\right)$ and $\tau\left(\Gamma_{\text {III,dec }}\right)$ are the lower and upper bounds of the twist $\tau$. The claim is true for type I and type III edgepath systems by Proposition 3.2. For a type II edgepath system $\Gamma$, by preparing $\Gamma^{\prime}$ as in the proof of the previous lemma, we easily have $\tau(\Gamma)=\tau\left(\Gamma^{\prime}\right)$ and $\tau\left(\Gamma_{\text {III,inc }}\right) \leq \tau\left(\Gamma^{\prime}\right) \leq \tau\left(\Gamma_{\text {III,dec }}\right)$ by an argument similar to Proposition 3.2. Hence, the twist is bounded by $\tau\left(\Gamma_{\text {III,inc }}\right)$ and $\tau\left(\Gamma_{\text {III,dec }}\right)$ also for any type II edgepath system. Thus,

$$
\left|\tau\left(\Gamma_{\text {III,inc }}\right)-\tau\left(\Gamma_{\text {III,dec }}\right)\right| \geq \operatorname{Diam}(K)
$$

Then, we have

$$
2 \operatorname{cr}(K) \geq \operatorname{Diam}(K)
$$

Finally, combining two-bridge cases in [10], Proposition 2.1, Lemmas 3.4 and 3.5, we have Theorem 1.1.

\section{Acknowledgment}

The first author was supported in part by grant-in-aid for Young Scientists (B) (no. 18740038).

\section{References}

[1] R.J. Aumann, Asphericity of alternating knots, Ann. Math. 64(2) (1956), 374-392.

[2] M. Culler and P.B. Shalen, Bounded, separating, incompressible surfaces in knot manifolds, Invent. Math. 75 (1984), 537-545. 
[3] C. Delman and R. Roberts, Alternating knots satisfy property $P$, Comment. Math. Helv. 74 (1999), 376-397.

[4] A.E. Hatcher, On the boundary curves of incompressible surfaces, Pacific J. Math. 99 (1982), 373-377.

[5] A.E. Hatcher and U. Oertel, Boundary slopes for Montesinos knots, Topology 28(4) (1989), 453-480.

[6] A.E. Hatcher and W.P. Thurston, Incompressible surfaces in 2-bridge knot complements, Invent. Math. 79 (1985), 225-246.

[7] K. Ichihara and S. Mizushima, Bounds on numerical boundary slopes for Montesinos knots, Hiroshima Math. J. 37(2) (2007), 211-252.

[8] W.B.R. Lickorish, An introduction to knot theory, Graduate texts in mathematics, New York, Springer, 1997, 175 pp.

[9] W.B.R. Lickorish and M.B. Thistlethwaite, Some links with non-trivial polynomials and their crossing-numbers, Comment. Math. Helv. 63 (1988), 527-539.

[10] T.W. Mattman, G. Maybrun and K. Robinson, 2-Bridge knot boundary slopes: diameter and genus, Osaka J. Math. 45(2) (2008), preprint version available at arXiv:math.GT/0510496.

College of General Education

OSAKA SANGYO UNIVERSITY

3-1-1 NaKagaito, DAITO

OSAKA 574-8530

JAPAN

Current address: Department of Mathematics Education

NARA UNIVERSITY OF EDUCATION

TAKABATAKE-CHO

NARA $630-8528$

JAPAN

E-mail address: ichihara@nara-edu.ac.jp

Department of Mathematical and Computing Sciences

Tokyo Institute of Technology

2-12-1 Ohokayama, Meguro

TOKYO 152-8552

JAPAN

E-mail address: mizusima@is.titech.ac.jp

Received July 17, 2006 
